MITSUBISHI ELECTRIC RESEARCH LABORATORIES

http://www.merl.com

\title{
Optimal and Suboptimal Linear Receivers for Time-Hopping Impulse Radio Systems
}

\author{
Sinan Gezici, Hisashi Kbayashi, H. Vincent Poor, Andreas F. Molisch
}

TR2004-052 May 2004

\begin{abstract}
In time-hopping impulse radio, a number of frames are allocated for each information symbol. In each of these frames, one ultra-wideband pulse is transmitted. During demodulation of the received signal, these pulses need to be optimally combined in order to achieve the lowest bit error probability. for a single-user system over an additive white Gaussian noise channel, an optimal linear scheme is the one in which samples from the received pulses in different frames are added with equal weight. However, in multiuser and/or frequency-selective environments, the contributions from different frames should be combined considering the minimum mean square error (MMSE) criterion in order to obtain low bit error rates. Moreover, in frequency-selective environments, where the receiver obtains samples from different multipaths, those multipath components should also be combined optimally. In this paper, we consider optimal and suboptimal linear receivers for a given user in a frequency-selective multiuser environment. The optimal linear receiver combining all the samples from the frames and the multipath components, according to the MMSE criterion is designed. Due to the complexity of this receiver, two suboptimal receivers are considered: i) An optimal frame combining receiver, which optimally combines the samples from the frames, while combing different multipath components suboptimally. ii) An optimal multipath combining receiver, which combines the samples from different multipath components optimally, while combining the samples from the frames suboptimally. In this paper, these optimal and suboptimal linear receivers are designed and their performance is evaluated via simulations.
\end{abstract}

UWBST 2004

This work may not be copied or reproduced in whole or in part for any commercial purpose. Permission to copy in whole or in part without payment of fee is granted for nonprofit educational and research purposes provided that all such whole or partial copies include the following: a notice that such copying is by permission of Mitsubishi Electric Research Laboratories, Inc.; an acknowledgment of the authors and individual contributions to the work; and all applicable portions of the copyright notice. Copying, reproduction, or republishing for any other purpose shall require a license with payment of fee to Mitsubishi Electric Research Laboratories, Inc. All rights reserved.

Copyright (C) Mitsubishi Electric Research Laboratories, Inc., 2004

201 Broadway, Cambridge, Massachusetts 02139 



\section{Optimal and Suboptimal Linear Receivers for Time-Hopping Impulse Radio Systems ${ }^{1}$}

\author{
Sinan Gezici, Hisashi Kobayashi and H. Vincent Poor ${ }^{1}$ \\ Department of Electrical Engineering \\ Princeton University, Princeton, NJ 08544 \\ \{sgezici,hisashi,poor\}@ princeton.edu
}

\author{
Andreas F. Molisch ${ }^{2}$ \\ Mitsubishi Electric Research Laboratories \\ 201 Broadway, Cambridge, MA 02139 \\ Andreas.Molisch@ieee.org
}

\begin{abstract}
In time-hopping impulse radio, a number of frames are allocated for each information symbol. In each of these frames, one ultra-wideband pulse is transmitted. During demodulation of the received signal, these pulses need to be optimally combined in order to achieve the lowest bit error probability. For a single-user system over an additive white Gaussian noise channel, an optimal linear scheme is the one in which samples from the received pulses in different frames are added with equal weight However, in multiuser and/or frequency-selective environments, the contributions from different frames should be combined considering the minimum mean square error (MMSE) criterion in order to obtain low bit error rates. Moreover, in frequency-selective environments, where the receiver obtains samples from different multipaths, those multipath components should also be combined optimally. In this paper, we consider optimal and suboptimal linear receivers for a given user in a frequency-selective multiuser environment. The optimal linear receiver combining all the samples from the frames and the multipath components, according to the MMSE criterion is designed. Due to the complexity of this receiver, two suboptimal receivers are considered: i) An optimal frame combining receiver, which optimally combines the samples from the frames, while combining different multipath components suboptimally. ii) An optimal multipath combining receiver, which combines the samples from different multipath components optimally, while combining the samples from the frames suboptimally. In this paper, these optimal and suboptimal linear receivers are designed and their performance is evaluated via simulations.
\end{abstract}

\section{INTRODUCTION}

Recently, communication systems that employ ultrawideband (UWB) signals have drawn considerable attention. UWB systems occupy a bandwidth larger than $500 \mathrm{MHz}$; due to the large spreading factors and low power spectral density, they can coexist with incumbent systems in the same frequency range. The recent Federal Communications Commission (FCC) rulings ([1], [2]) specify the regulations for UWB communication systems in the US. Similar rulings are expected in the near future in Europe and Japan as well.

\footnotetext{
${ }^{1}$ This research is supported in part by the National Science Foundation under grant CCR-99-79361, and in part by the New Jersey Center for Wireless Telecommunications.

${ }^{2}$ Also at the Department of Electroscience, Lund University, Lund, Sweden.
}

Impulse radio (IR) systems, which transmit very short pulses with a low duty cycle, are typically employed to implement UWB systems ([3]-[5]). In an IR system, a number of frames are allocated to each information symbol. In each frame, a UWB pulse is transmitted and its position in the frame is determined by a time-hopping (TH) sequence [3].

The number of frames/pulses that are sent per information symbol is denoted by $N_{f}$. In a single user system over an additive white Gaussian noise (AWGN) channel, the received signal consists of $N_{f}$ pulses in $N_{f}$ frames. After matched-filtering and sampling, the contributions from the frames are added with equal weight to form the decision variable [3]. In considering a multiuser environment, the contributions from different frames can have different signal-to-interference-plus-noise ratios (SINR) depending on the TH sequences of the users. Therefore, equally-weighted contributions from the different frames no longer form an optimal decision variable. Also, in a frequency-selective environment, there can be selfinterference, also called inter-frame interference (IFI), due to multipath, which affects the optimal combining of the frame components at the receiver.

Apart from different contributions from $N_{f}$ frames, there is also diversity due to the frequency-selective environment. Optimal combination of different multipath components is affected by multiple access interference (MAI) and IFI. In other words, we need to consider the optimal combination of contributions from both $N_{f}$ different frames and the different multipath components.

In this paper, we first consider the optimal linear receiver for a given user in the frequency-selective multiuser environment, which combines all the samples from the received signal according to the minimum mean square error (MMSE) criterion. Due to the complexity of this optimal receiver, we also consider two suboptimal receivers with lower complexity. The first receiver is called an optimal frame combining (OFC) receiver, which combines the samples from different frames according to the MMSE criterion and combines the samples from different multipath components according to the maximal ratio combining (MRC) scheme. The other receiver is called an optimal multipath combining (OMC) receiver, which combines the contribu- 


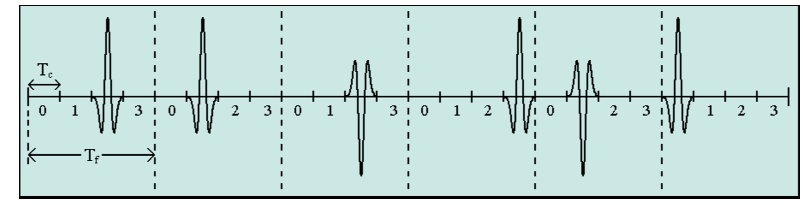

Fig. 1. An example time-hopping impulse radio signal with pulsebased polarity randomization, where $N_{f}=6, N_{c}=4$, the time hopping sequence is $\{2,1,2,3,1,0\}$ and the polarity codes are $\{+1,+1,-$ $1,+1,-1,+1\}$.

tions from different multipath components optimally, while combining the contributions from different frames suboptimally.

The remainder of the paper is organized as follows. Section 2 describes the signal model for an IR system and presents a discrete-time representation of the received signal. Section 3 investigates the optimal linear receiver that combines all the components of the received signal according to the MMSE criterion. The OFC and the OMC receivers are presented in Section 4 and Section 5, respectively. After simulation results are presented in Section 6, some conclusions are drawn in Section 7.

\section{SignAl MODEL}

We consider a synchronous, binary phase shift keyed TH-IR system with $K$ users, in which the transmitted signal from user $k$ is represented by:

$s_{t x}^{(k)}(t)=\sqrt{\frac{E_{k}}{N_{f}}} \sum_{j=-\infty}^{\infty} d_{j}^{(k)} b_{\left\lfloor j / N_{f}\right\rfloor}^{(k)} p_{t x}\left(t-j T_{f}-c_{j}^{(k)} T_{c}\right)$,

where $p_{t x}(t)$ is the transmitted UWB pulse, $E_{k}$ is the bit energy of user $k, T_{f}$ is the average pulse repetition time (also called the "frame" time), $N_{f}$ is the number of pulses representing one information symbol, and $b_{\left\lfloor j / N_{f}\right\rfloor}^{(k)} \in\{+1,-1\}$ is the binary information symbol transmitted by user $k$. In order to allow the channel to be shared by many users and avoid catastrophic collisions, a time-hopping (TH) sequence $\left\{c_{j}^{(k)}\right\}$, where $c_{j}^{(k)} \in\left\{0,1, \ldots, N_{c}-1\right\}$, is assigned to each user. This TH sequence provides an additional time shift of $c_{j}^{(k)} T_{c}$ seconds to the $j$ th pulse of the $k$ th user where $T_{c}$ is the chip interval and is chosen to satisfy $T_{c} \leq T_{f} / N_{c}$ in order to prevent the pulses from overlapping. The random polarity codes $d_{j}^{(k)}$ are binary random variables taking values \pm 1 with equal probability ([6]-[8]).

Assuming a tapped-delay-line channel model with multipath resolution $T_{c}$, the discrete channel model $\boldsymbol{\alpha}^{(k)}=\left[\alpha_{1}^{(k)} \cdots \alpha_{L}^{(k)}\right]$ is adopted for user $k$, where $L$ is assumed, without loss of generality, to be the number of multipath components for each user. Then, the received

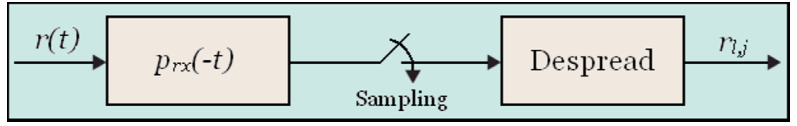

Fig. 2. Match-filtering, sampling and despreading of the received signal.

signal can be expressed as

$$
\begin{aligned}
& r(t)=\sum_{k=1}^{K} \sqrt{\frac{E_{k}}{N_{f}}} \sum_{j=-\infty}^{\infty} \sum_{l=1}^{L} \alpha_{l}^{(k)} d_{j}^{(k)} b_{\left\lfloor j / N_{f}\right\rfloor}^{(k)} \\
& \times p_{r x}\left(t-j T_{f}-c_{j}^{(k)} T_{c}-(l-1) T_{c}\right)+\sigma_{n} n(t),
\end{aligned}
$$

where $p_{r x}(t)$ is the received unit-energy UWB pulse, and $n(t)$ is a zero mean white Gaussian noise with unit spectral density.

Consider a filter matched to the UWB pulse $p_{r x}(t)$, as shown in Figure 2. The output of this filter is sampled at instants when the paths $l \in \mathcal{L}$ arrive in each frame, where $\mathcal{L}=\left\{l_{1}, \ldots, l_{M}\right\}$ with $M \leq L$. Due to possible collisions, the actual number $N$ of total samples per information symbol can be smaller than $N_{f} M$. The samples at the output of the matched filter are despread ${ }^{3}$ by the polarity code of a user of interest, say user 1 (Figure 2).

The discrete signal at the $l$ th path of the $j$ th frame can then be expressed, for the $i$ th information bit, as ${ }^{4}$

$$
r_{l, j}=\mathbf{s}_{l, j}^{T} \mathbf{A} \mathbf{b}_{i}+n_{l, j},
$$

for $l=l_{1}, \ldots, l_{M}$ and $j=i N_{f}, \ldots,(i+1) N_{f}-1$, where $\mathbf{A}=\operatorname{diag}\left\{\sqrt{E_{1} / N_{f}}, \ldots, \sqrt{E_{K} / N_{f}}\right\}, \mathbf{b}_{i}=$ $\left[b_{i}^{(1)} \cdots b_{i}^{(K)}\right]^{T}$ and $n_{l, j} \sim \mathcal{N}\left(0, \sigma_{n}^{2}\right) . \mathbf{s}_{l, j}$ is a $K \times 1$ vector, which can be expressed as a sum of the desired signal part (SP), inter-frame interference (IFI) and multiple-access interference (MAI) terms:

$$
\mathbf{s}_{l, j}=\mathbf{s}_{l, j}^{(S P)}+\mathbf{s}_{l, j}^{(I F I)}+\mathbf{s}_{l, j}^{(M A I)}
$$

where the $k$ th elements can be expressed as

$$
\begin{aligned}
{\left[\mathbf{s}_{l, j}^{(S P)}\right]_{k} } & = \begin{cases}\alpha_{l}^{(1)}, & k=1 \\
0, & k=2, \ldots, K\end{cases} \\
{\left[\mathbf{s}_{l, j}^{(I F I)}\right]_{k} } & = \begin{cases}d_{j}^{(1)} \sum_{(n, m) \in \mathcal{A}_{l, j}} d_{m}^{(1)} \alpha_{n}^{(1)}, & k=1 \\
0, & k=2, \ldots, K\end{cases} \\
{\left[\mathbf{s}_{l, j}^{(M A I)}\right]_{k} } & = \begin{cases}0, & k=1 \\
d_{j}^{(1)} \sum_{(n, m) \in \mathcal{B}_{l, j}^{(k)}} d_{m}^{(k)} \alpha_{n}^{(k)}, & k=2, \ldots, K\end{cases}
\end{aligned}
$$

\footnotetext{
${ }^{3}$ In the context of IR systems, spreading and despreading by random polarity codes are not intended for expanding the bandwidth of the signal. It mainly helps reduce the effect of MAI [6] and eliminate the spectral lines [8].

${ }^{4}$ Note that the dependence of $r_{l, j}$ on the index of the information bit, $i$, is not shown explicitly.
} 
with

$$
\begin{gathered}
\mathcal{A}_{l, j}=\left\{(n, m): n \in\{1, \ldots, L\}, m \in \mathcal{F}_{i}, m \neq j,\right. \\
\left.m T_{f}+c_{m}^{(1)} T_{c}+n T_{c}=j T_{f}+c_{j}^{(1)} T_{c}+l T_{c}\right\}
\end{gathered}
$$

and

$$
\begin{aligned}
& \mathcal{B}_{l, j}^{(k)}=\left\{(n, m): n \in\{1, \ldots, L\}, m \in \mathcal{F}_{i},\right. \\
& \left.m T_{f}+c_{m}^{(k)} T_{c}+n T_{c}=j T_{f}+c_{j}^{(1)} T_{c}+l T_{c}\right\}
\end{aligned}
$$

where $\mathcal{F}_{i}=\left\{i N_{f}, \ldots,(i+1) N_{f}-1\right\}$.

Note that $\mathcal{A}_{l, j}$ is the set of frame and multipath indices of pulses from user 1 that originate from a frame different from the $j$ th one and collide with the $l$ th path of the $j$ th pulse of user 1 . Similarly, $\mathcal{B}_{l, j}^{(k)}$ is the set of frame and path indices of pulses from user $k$ that collide with the $l$ th path of the $j$ th pulse of user 1 .

For simplicity of the analysis, we assume a guard interval between information symbols that is equal to the length of the channel impulse response (e.g. [9]), which avoids inter-symbol interference (ISI). Therefore, for bit $i$, we only consider the interference from the pulses in the frames of the current symbol $i$, namely, from the pulses in frames $i N_{f}, \ldots,(i+1) N_{f}-1$.

\section{LINEAR MMSE RECEIVER}

In this section, we consider a linear receiver for user 1 that combines all the samples from the received signal optimally, according to the MMSE criterion.

Let $\mathbf{r}$ be an $N \times 1$ vector denoting the distinct samples $r_{l, j}$ for $(l, j) \in \mathcal{L} \times\left\{1, \ldots, N_{f}\right\}$ :

$$
\mathbf{r}=\left[r_{l_{1}, j_{1}^{(1)}} \cdots r_{l_{1}, j_{m_{1}}^{(1)}} \cdots r_{l_{M}, j_{1}^{(M)}} \cdots r_{l_{M}, j_{m_{M}}^{(M)}}\right]^{T}
$$

where $\sum_{i=1}^{M} m_{i}=N$ denotes the total number of samples, with $N \leq M N_{f}$.

Using (3), $\mathbf{r}$ can be expressed as

$$
\mathbf{r}=\mathbf{S A} \mathbf{b}_{i}+\mathbf{n},
$$

where $\mathbf{A}$ and $\mathbf{b}_{i}$ are as in (3) and $\mathbf{n} \sim \mathcal{N}\left(\mathbf{0}, \sigma_{n}^{2} \mathbf{I}\right)$. $\mathbf{S}$ is a signature matrix, which has $\mathbf{s}_{l, j}^{T}$ (see (4) through (7)) for $(l, j) \in \mathcal{C}$ as its rows, where $\mathcal{C}=$ $\left\{\left(l_{1}, j_{1}^{(1)}\right), \ldots,\left(l_{1}, j_{m_{1}}^{(1)}\right), \ldots,\left(l_{M}, j_{1}^{(M)}\right), \ldots,\left(l_{M}, j_{m_{M}}^{(M)}\right)\right\}$.

From (4)-(7), $\mathbf{S}$ can be expressed as $\mathbf{S}=\mathbf{S}^{(S P)}+$ $\mathbf{S}^{(I F I)}+\mathbf{S}^{(M A I)}$. Then, after some manipulations, $\mathbf{r}$ becomes

$$
\mathbf{r}=b_{i}^{(1)} \sqrt{\frac{E_{1}}{N_{f}}}(\boldsymbol{\alpha}+\mathbf{e})+\mathbf{S}^{(M A I)} \mathbf{A} \mathbf{b}_{i}+\mathbf{n},
$$

where $\boldsymbol{\alpha}=\left[\alpha_{l_{1}}^{(1)} \mathbf{1}_{m_{1}}^{T} \cdots \alpha_{l_{M}}^{(1)} \mathbf{1}_{m_{M}}^{T}\right]^{T}$, with $\mathbf{1}_{m}$ denoting an $m \times 1$ vector of all ones, and $\mathbf{e}$ is an $N \times 1$ vector whose elements are $e_{l, j}=d_{j}^{(1)} \sum_{(n, m) \in \mathcal{A}_{l, j}} d_{m}^{(1)} \alpha_{n}^{(1)}$ for $(l, j) \in \mathcal{C}$.

A linear receiver combines the elements of $\mathbf{r}$ and obtains a decision variable as follows:

$$
y_{1}=\boldsymbol{\theta}^{T} \mathbf{r}
$$

where $\boldsymbol{\theta}$ is the weighting vector.

The MMSE weights that maximize the SINR of the received signal in (12) can be obtained [10] as

$$
\boldsymbol{\theta}_{M M S E}=\mathbf{R}_{\mathbf{w}_{1}}^{-1}(\boldsymbol{\alpha}+\mathbf{e})
$$

where $\mathbf{w}_{1}=\mathbf{S}^{(M A I)} \mathbf{A} \mathbf{b}_{i}+\mathbf{n}$ and $\mathbf{R}_{\mathbf{w}_{1}}=\mathrm{E}\left\{\mathbf{w}_{1} \mathbf{w}_{1}^{T}\right\}$. Assuming equiprobable information symbols, the correlation matrix can be expressed as

$$
\mathbf{R}_{\mathbf{w}_{1}}=\mathbf{S}^{(M A I)} \mathbf{A}^{2}\left(\mathbf{S}^{(M A I)}\right)^{T}+\sigma_{n}^{2} \mathbf{I} .
$$

Then, the linear MMSE receiver becomes

$$
\hat{b}_{i}^{(1)}=\operatorname{sign}\left\{\mathbf{r}^{T} \mathbf{R}_{\mathbf{w}_{1}}^{-1}(\boldsymbol{\alpha}+\mathbf{e})\right\} .
$$

Note that this receiver requires the inversion of an $N \times N$ matrix $\left(N \leq M N_{f}\right)$. Hence, it can be very complex in some situations. Therefore, we investigate some suboptimal linear receivers in the following subsections.

\section{Optimal Frame Combining (OFC)}

In this case, the multipath components in each frame are added according to the MRC criterion. Then, those combined components in the frames are combined according to the MMSE criterion. That is, the decision variable is given by

$$
y_{2}=\sum_{j=i N_{f}}^{(i+1) N_{f}-1} \gamma_{j} \sum_{l \in \mathcal{L}} \alpha_{l}^{(1)} r_{l, j},
$$

where $\gamma_{i N_{f}}, \ldots, \gamma_{(i+1) N_{f}-1}$ are the weighting factors for the $i$ th bit.

From (3), $y_{2}$ can be expressed as

$$
y_{2}=\gamma_{i}^{T}\left(\sum_{l \in \mathcal{L}} \alpha_{l}^{(1)} \hat{\mathbf{S}}_{l} \mathbf{A} \mathbf{b}_{i}+\sum_{l \in \mathcal{L}} \alpha_{l}^{(1)} \hat{\mathbf{n}}_{l}\right)
$$

where $\gamma_{i}=\left[\gamma_{i N_{f}} \cdots \gamma_{(i+1) N_{f}-1}\right]^{T}$ is the vector of weighting coefficients, $\hat{\mathbf{n}}_{l}=\left[n_{l, i N_{f}} \cdots n_{l,(i+1) N_{f}-1}\right]^{T}$ is the noise vector, which is distributed as $\mathcal{N}\left(\mathbf{0}, \sigma_{n}^{2} \mathbf{I}\right)$, and $\hat{\mathbf{S}}_{l}$ is an $N_{f} \times K$ matrix, whose $j$ th row is $\mathbf{s}_{l, i N_{f}+j-1}^{T}$. Using (4)-(7), $\hat{\mathbf{S}}$ can be expressed as $\hat{\mathbf{S}}=$ $\hat{\mathbf{S}}^{(S P)}+\hat{\mathbf{S}}^{(I F I)}+\hat{\mathbf{S}}^{(M A I)}$. Then, we get

$y_{2}=\gamma_{i}^{T}\left(b_{i}^{(1)} \sqrt{\frac{E_{1}}{N_{f}}}\left[\sum_{l \in \mathcal{L}}\left(\alpha_{l}^{(1)}\right)^{2} \mathbf{1}_{N_{f}}+\sum_{l \in \mathcal{L}} \alpha_{l}^{(1)} \hat{\mathbf{e}}_{l}\right]+\mathbf{w}_{2}\right)$,

where $\hat{\mathbf{e}}_{l}$ is an $N_{f} \times 1$ vector whose $j$ th element is $e_{l, i N_{f}+j-1}=d_{i N_{f}+j-1}^{(1)} \sum_{(n, m) \in \mathcal{A}_{l, i N_{f}+j-1}} d_{m}^{(1)} \alpha_{n}^{(1)}$ and $\mathbf{w}_{2}=\sum_{l \in \mathcal{L}} \alpha_{l}^{(1)} \hat{\mathbf{S}}_{l}^{(M A I)} \mathbf{A} \mathbf{b}_{i}+\sum_{l \in \mathcal{L}} \alpha_{l}^{(1)} \hat{\mathbf{n}}_{l}$.

From (19), the MMSE weights can be obtained as

$$
\gamma_{M M S E}=\mathbf{R}_{\mathbf{w}_{2}}^{-1}\left(\sum_{l \in \mathcal{L}}\left(\alpha_{l}^{(1)}\right)^{2} \mathbf{1}_{N_{f}}+\sum_{l \in \mathcal{L}} \alpha_{l}^{(1)} \hat{\mathbf{e}}_{l}\right)
$$


where

$$
\begin{aligned}
\mathbf{R}_{\mathbf{w}_{2}} & =\sum_{l \in \mathcal{L}} \alpha_{l}^{(1)} \hat{\mathbf{S}}_{l}^{(M A I)} \mathbf{A}^{2} \sum_{l \in \mathcal{L}} \alpha_{l}^{(1)}\left(\hat{\mathbf{S}}_{l}^{(M A I)}\right)^{T} \\
& +\sum_{l_{1} \in \mathcal{L}} \sum_{l_{2} \in \mathcal{L}} \alpha_{l_{1}}^{(1)} \alpha_{l_{2}}^{(1)} \mathrm{E}\left\{\hat{\mathbf{n}}_{l_{1}} \hat{\mathbf{n}}_{l_{2}}^{T}\right\}
\end{aligned}
$$

It is straightforward to show that $\mathrm{E}\left\{\hat{\mathbf{n}}_{l_{1}} \hat{\mathbf{n}}_{l_{2}}^{T}\right\}=\sigma_{n}^{2} \mathbf{I}$ for $l_{1}=l_{2}$. When $l_{1} \neq l_{2}$, the element at row $j_{1}$ and column $j_{2},\left[\mathrm{E}\left\{\hat{\mathbf{n}}_{l_{1}} \hat{\mathbf{n}}_{l_{2}}^{T}\right\}\right]_{j_{1} j_{2}}$, is equal to $\sigma_{n}^{2}$ if $j_{1} N_{c}+$ $c_{j_{1}}^{(1)}+l_{1}=j_{2} N_{c}+c_{j_{2}}^{(1)}+l_{2}$ and zero otherwise $\left(j_{1}=\right.$ $i N_{f}, \ldots,(i+1) N_{f}-1$ and $\left.j_{2}=i N_{f}, \ldots,(i+1) N_{f}-1\right)$.

We note from (19) and (20) that the OFC receiver, $\hat{b}_{i}^{(1)}=\operatorname{sign}\left\{y_{2}\right\}$, requires the inversion of an $N_{f} \times N_{f}$ matrix. The reduction in complexity compared to the optimal linear MMSE receiver of the previous section is due to the suboptimal combination of the multipath components.

The SINR of the system can be expressed as

$$
S I N R_{O F C}=\frac{E_{1}}{N_{f}} \mathbf{x}_{2}^{T} \mathbf{R}_{\mathbf{w}_{2}}^{-1} \mathbf{x}_{2} .
$$

where $\mathbf{x}_{2}=\sum_{l \in \mathcal{L}}\left(\alpha_{l}^{(1)}\right)^{2} \mathbf{1}_{N_{f}}+\sum_{l \in \mathcal{L}} \alpha_{l}^{(1)} \hat{\mathbf{e}}_{l}$.

\section{Optimal Multipath Combining (OMC)}

Now consider a receiver that combines different multipath components optimally, according to the MMSE criterion, while employing equal gain combining (EGC) for contributions from different frames. In this case, the decision variable is given by

$$
y_{3}=\sum_{l \in \mathcal{L}} \beta_{l} \sum_{j=i N_{f}}^{(i+1) N_{f}-1} r_{l, j},
$$

where $\boldsymbol{\beta}=\left[\beta_{l_{1}} \cdots \beta_{l_{M}}\right]^{T}$ is the weighting vector.

Using (3), $y_{3}$ can be expressed as

$$
y_{3}=\boldsymbol{\beta}^{T}\left(\sum_{j=i N_{f}}^{(i+1) N_{f}-1}\left[\tilde{\mathbf{S}}_{j} \mathbf{A} \mathbf{b}_{i}+\tilde{\mathbf{n}}_{j}\right]\right) \text {, }
$$

where $\tilde{\mathbf{n}}_{j}=\left[n_{l_{1}, j} \cdots n_{l_{M}, j}\right]^{T}$ is the noise vector, which is distributed as $\mathcal{N}\left(\mathbf{0}, \sigma_{n}^{2} \mathbf{I}\right)$ and $\tilde{\mathbf{S}}_{j}$ is an $M \times K$ signature matrix, whose $m$ th row is $\mathbf{s}_{l_{m}, j}^{T}$. Using (4)-(7), $\tilde{\mathbf{S}}$ can be expressed as $\tilde{\mathbf{S}}=\tilde{\mathbf{S}}^{(S P)}+\tilde{\mathbf{S}}^{(I F I)}+\tilde{\mathbf{S}}^{(M A I)}$. Then, we get

$$
y_{3}=\boldsymbol{\beta}^{T}\left[b_{i}^{(1)} \sqrt{\frac{E_{1}}{N_{f}}}\left(N_{f} \tilde{\boldsymbol{\alpha}}+\sum_{j=i N_{f}}^{(i+1) N_{f}-1} \tilde{\mathbf{e}}_{j}\right)+\mathbf{w}_{3}\right] \text {, }
$$

where $\tilde{\boldsymbol{\alpha}}=\left[\alpha_{l_{1}}^{(1)} \cdots \alpha_{l_{M}}^{(1)}\right]^{T}, \tilde{\mathbf{e}}_{j}$ is an $M \times 1$ vector whose $m$ th element is $e_{l_{m}, j}=d_{j}^{(1)} \sum_{(n, m) \in \mathcal{A}_{l_{m}, j}} d_{m}^{(1)} \alpha_{n}^{(1)}$, and $\mathbf{w}_{3}=\sum_{j=i N_{f}}^{(i+1) N_{f}-1} \tilde{\mathbf{S}}_{j}^{(M A I)} \mathbf{A} \mathbf{b}_{i}+\sum_{j=i N_{f}}^{(i+1) N_{f}-1} \tilde{\mathbf{n}}_{j}$.
From (25), the MMSE weights are chosen as

$$
\boldsymbol{\beta}_{M M S E}=\mathbf{R}_{\mathbf{w}_{3}}^{-1}\left(N_{f} \tilde{\boldsymbol{\alpha}}+\sum_{j=i N_{f}}^{(i+1) N_{f}-1} \tilde{\mathbf{e}}_{j}\right),
$$

where

$$
\begin{aligned}
\mathbf{R}_{\mathbf{w}_{3}} & =\sum_{j=i N_{f}}^{(i+1) N_{f}-1} \tilde{\mathbf{S}}_{j}^{(M A I)} \mathbf{A}^{2} \sum_{j=i N_{f}}^{(i+1) N_{f}-1}\left(\tilde{\mathbf{S}}_{j}^{(M A I)}\right)^{T} \\
& +\sum_{j_{1}=i N_{f}}^{(i+1) N_{f}-1} \sum_{j_{2}=i N_{f}}^{(i+1) N_{f}-1} \mathrm{E}\left\{\tilde{\mathbf{n}}_{j_{1}} \tilde{\mathbf{n}}_{j_{2}}^{T}\right\}
\end{aligned}
$$

It can be observed that $\mathrm{E}\left\{\tilde{\mathbf{n}}_{j_{1}} \tilde{\mathbf{n}}_{j_{2}}^{T}\right\}=\sigma_{n}^{2} \mathbf{I}$ for $j_{1}=j_{2}$. When $j_{1} \neq j_{2}$, the element at row $l_{1}$ and column $l_{2}$, $\left[\mathrm{E}\left\{\hat{\mathbf{n}}_{j_{1}} \hat{\mathbf{n}}_{j_{2}}^{T}\right\}\right]_{l_{1} l_{2}}$, is equal to $\sigma_{n}^{2}$ if $l_{1} N_{c}+c_{l_{1}}^{(1)}+j_{1}=$ $l_{2} N_{c}+c_{l_{2}}^{(1)}+j_{2}$ and zero otherwise $\left(l_{1} \in \mathcal{L}\right.$ and $\left.l_{2} \in \mathcal{L}\right)$.

We note from (25) and (26) that the OMC receiver, $\hat{b}_{i}^{(1)}=\operatorname{sign}\left\{y_{3}\right\}$, needs to invert the $M \times M$ matrix $\mathbf{R}_{\mathbf{w}_{3}}$. The reduction in the complexity compared to the optimal linear receiver in Section 3 is the result of suboptimal combination of the contributions from different frames.

The SINR of the system can be expressed as

$$
S I N R_{O M C}=\frac{E_{1}}{N_{f}} \mathbf{x}_{3}^{T} \mathbf{R}_{\mathbf{w}_{3}}^{-1} \mathbf{x}_{3} .
$$

where $x_{3}=N_{f} \tilde{\boldsymbol{\alpha}}+\sum_{j=i N_{f}}^{(i+1) N_{f}-1} \tilde{\mathbf{e}}_{j}$.

From the previous equations, the following property follows:

Property: Consider a single user system where the pulses in a frame never collide with any pulse in another frame. In other words, there is no IFI and MAI. In this case, the expressions for SINR can be shown to reduce to

$$
S I N R_{O F C}=S I N R_{O M C}=\frac{E_{1}}{\sigma_{n}^{2}} \sum_{l \in \mathcal{L}} \alpha_{l}^{2} .
$$

\section{Simulation Results}

In this section, we consider the downlink of a THIR system with 5 users $(K=5)$, where $E_{k}=1$ $\forall k$. The number of chips per frame, $N_{c}$, is equal to 10 and the discrete channel is given by $\boldsymbol{\alpha}^{(k)}=$

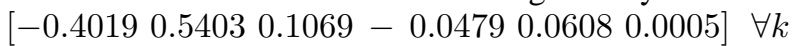
[11]. The TH sequences and polarity codes of the users are chosen from appropriate uniform distributions, and the results are averaged over different realizations.

In the first scenario, the number of frames per symbol, $N_{f}$, is equal to 10 and the first three multipaths are sampled at the receiver; that is, $\mathcal{L}=\{1,2,3\}$. Figure 3 shows the bit error probability (BEP) for different SNR values. From the figure, it is observed that the optimal linear receiver performs the best as expected. The OFC receiver performs better than the OMC receiver in this case, which implies that there is a greater diversity gain in combining 10 different frames than combining 3 


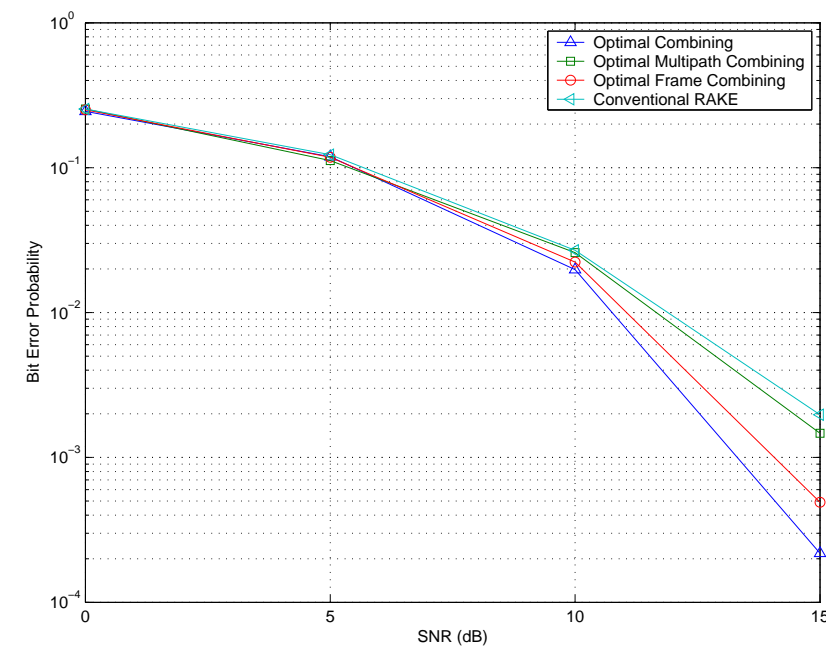

Fig. 3. BEP versus SNR for a 5-user TH-IR over the channel

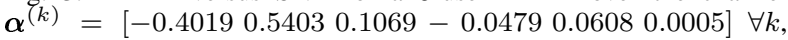
where $N_{c}=10$ and $E_{k}=1 \forall k$. The number of frames per information bit, $N_{f}$, is 10 and the first three paths are combined at the receiver.

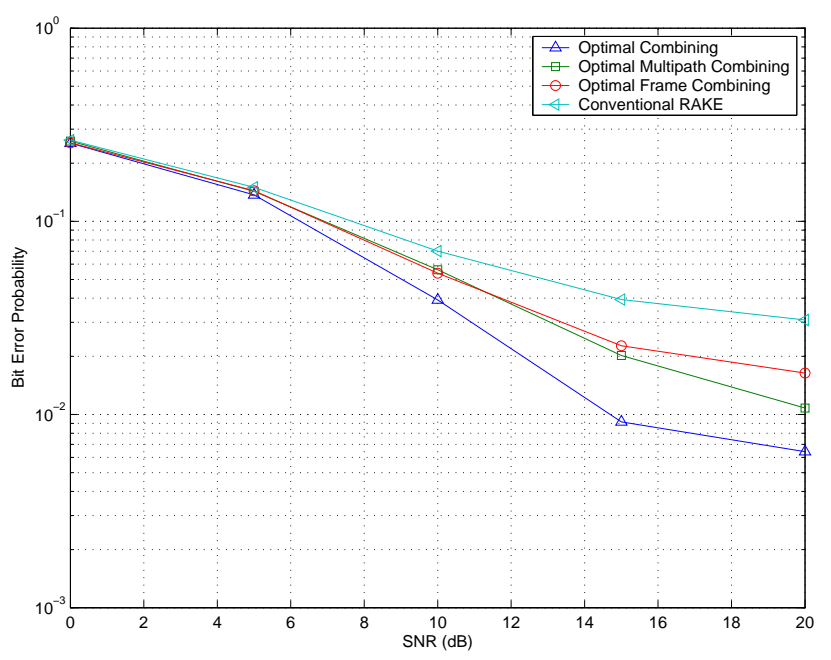

Fig. 4. BEP versus SNR for a 5-user TH-IR over the channel

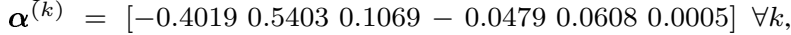
where $N_{c}=10$ and $E_{k}=1 \forall k$. The number of frames per information bit, $N_{f}$, is 2 and all the multipath components are combined at the receiver.

multipath components. Also note that the OFC receiver needs to invert a $10 \times 10$ matrix while the $\mathrm{OMC}$ receiver needs the inversion of a $3 \times 3$ matrix. Finally, the conventional RAKE receiver, which performs EGC across the frames and MRC across the multipath components, has the highest BEP values due to its suboptimal combining schemes in both diversity domains.

In Figure $4, N_{f}=2$ and all the paths of the received signal are sampled; that is, $\mathcal{L}=\{1,2,3,4,5,6\}$. From the plot, it is observed that the optimal linear receiver is the best and the conventional RAKE is the worst, as expected. However, in this case, the OMC receiver performs better than the OFC receiver because $N_{f}$ is small in this case and optimally combining these two components is less important than the optimal combination of the six multipath components. Also note that the SNR is increased by decreasing the noise power. Hence, after some point, the BEP does not decrease much, since the errors are mainly due to MAI and IFI.

\section{CONCluding REMARKS}

We have considered optimal and suboptimal linear receivers for TH-IR systems. The optimal linear receiver performs MMSE combining of all the received samples. It gives the best BEP performance, but its complexity is usually very high. Therefore, we have proposed the OFC receiver, which combines the contributions from the frames optimally, while performing MRC for the received multipath components. Finally, we have considered the OMC receiver, which combines the components from different frames with equal weight while using the MMSE criterion for the multipath components. Depending on the system parameters, the OMC receiver could beat the OFC receiver and vice versa.

These receivers may not be very practical in real environments. However, they provide important theoretical references for more practical receivers. Furthermore, these receivers may be feasible, in downlinks of some TH-IR systems, where the base station (or the piconet coordinator) transmits information about the $\mathrm{TH}$ sequences and polarity codes of all the users.

\section{REFERENCES}

[1] FCC 00-163: Notice of Proposed Rule Making.

[2] FCC 02-48: First Report and Order.

[3] M. Z. Win and R. A. Scholtz, "Impulse radio: How it works," IEEE Communications Letters, 2(2): pp. 36-38, Feb. 1998.

[4] R. A. Scholtz, "Multiple access with time-hopping impulse modulation Scholtz," Proc. IEEE Military Communications Conference, 1993 (MILCOM'93), vol. 2, pp. 447-450, Bedford, MA, Oct. 1993.

[5] M. Z. Win and R. A. Scholtz, "Ultra-wide bandwidth timehopping spread-spectrum impulse radio for wireless multipleaccess communications," IEEE Trans. on Communications, vol. 48, issue 4, pp. 679-691, April 2000.

[6] E. Fishler and H. V. Poor, "On the tradeoff between two types of processing gain," 40th Annual Allerton Conference on Communication, Control, and Computing, Monticello, IL, Oct. 2-4, 2002

[7] S. Gezici, H. Kobayashi, H. V. Poor, and A. F. Molisch, "Performance Evaluation of Impulse Radio UWB Systems with Pulse-Based Polarity Randomization in Asynchronous Multiuser Environments," IEEE Wireless Communications and Networking Conference (WCNC'04), Atlanta, GA, March 2004.

[8] Y.-P. Nakache and A. F. Molisch, "Spectral shape of UWB signals influence of modulation format, multiple access scheme and pulse shape," Proceedings of the IEEE Vehicular Technology Conference, (VTC 2003-Spring), vol. 4, pp. 2510-2514, Jeju, Korea, April 2003.

[9] J. Evans and D. N. C. Tse, "Large System Performance of Linear Multiuser Receivers in Multipath Fading Channels," IEEE Trans. Inform. Theory, IT-46(6):2059-2078, September 2000.

[10] S. Verdú. Multiuser Detection, Cambridge University Press, Cambridge, UK, 1998.

[11] C. J. Le-Martret and G. B. Giannakis, "All-digital PAM impulse radio for multiple-access through frequency-selective multipath," Proceedings of the IEEE Global Telecommunications Conference (GLOBECOM 2000), vol. 1, pp. 77-81, San Fransisco, CA, Nov. 2000. 